\title{
CCTV Surveillance for Unprecedented Violence and Traffic Monitoring
}

\author{
Dr. R. Dhaya \\ Professor, \\ Dept. of Computer Science and Engineering, \\ King Khalid University, \\ Kindom of Saudi Arabia. \\ dhayavel2005@gmail.com
}

\begin{abstract}
Monitoring of traffic and unprecedented violence has become very much necessary in the urban as well as the rural areas, so the paper attempts to develop a CCTV surveillance for unprecedented violence and traffic monitoring. The proffered method performs the synchronization of the videos and does proper alliance employing the algorithms of motion detection and contour filtering. The steps in motion detection identifies the movement of the objects such as vehicles and unprecedented activities whereas the filtering is used to identify the object itself using its color. The synchronization and the alignment process affords to provide the details of the each objects on the scenario. The proposed algorithm is developed in Java which assists its model using its library that is open source. The validation of the proposed model was carried out using the data set acquired from real time and results were acquired. Moreover the results acquired were compared with the algorithms that were created in the early stages, the comparison proved that the proffered model was capable of obtaining a consecutive quick outcomes of $12.3912 *$ factor than the existing methods for the resolution of the video used in testing was 240.01 x 320.01 with 40 frames per second with cameras of high definition. Further the results acquired were computed to run the application of the embedded CPU and the GPU processors.
\end{abstract}

Keywords: CCTV Surveillance, Unprecedented Violence, Traffic Monitoring, Motion Detection, Contour Filtering

\section{Introduction}

Nowadays tracking and monitoring of human behaviors in the public has become one of the prominent area of research. The CCTV surveillance are capable empowering a broader range of application with diverse attributes. In order to improvise the technology of the surveillance system it essential that the system must be proficient of comprehending the happenings that are taking place. The comprehending process over the images and the video is an interdisciplinary area involving multiple computer vision procedures. The applications such as the surveillance cameras, diving assistance and the smart phones are some of the computer vision applications that are highly popular. Our proffered method also relies on the computational- intensive computer vision application to operate a hardware accelerator by developing the procedures to be followed for aligning the videos and identifying the changes in the environment. 
The videos captured in real time gathered and segregated into two categories as training and testing videos for the proposed model, the videos were captured on similar paths and the videos with the optimal pair is selected for training and testing. After finding out the optimal pairs the algorithms are applied over the videos to find out the dangerous happenings. The mechanism put forth is capable of performing multiple operations such as, identifying the disappeared objects unprecedented violence, traffic monitoring and assisting the driver in the traffic etc.

The issues in ordering the videos captured using a moving camera on similar path according to their geo position information is managed perfectly using the proffered mechanism. The aligning and the synchronization of the videos captured were initially developed using the datasets that were captured using the cameras that were fixed in a place. But was not good enough in providing accurate outcomes and was not aided with an open source model. So the paper attempts in providing a CCTV surveillance with an effective open source, alignment and synchronization of videos. The motion detection and contour filtering procedures are employed to identify the object movement and the object itself respectively.

The prevailing systems developed synchronization in the videos and ordered the videos by performing the comparison between the videos in the sequence and sorting out the temporal as well as the spatial correspondence among every frames of videos. Additionally the linear correspondence among the frames were also used and this imposed limitations in the earlier system making them in suitable for practical application. The low accuracy incurred in their processing made the prevailing systems them less efficient.

The proposed method of surveillance that aimed in developing an interface that is user friendly for video uploading, along with the implementation of the algorithm that is capable of matching low dimensional frames to order the videos, to provide a outcomes in a very efficient method is organized with related works, proposed system, results evaluation and conclusion in the section $2,3,4$, and 5 respectively.

\section{Related Works}

The section presents similar existing works carried out to have a proper surveillance and by solving the issue in ordering and synchronizing the videos, the methods used in the prevailing system compared the temporal and the spatial details of the video sequences as in Bradski, Gary et al. [1], but he synchronizing process in the initial stages were developed using the cameras that were fixed as in the method followed by the author Lowe, et al [2].

Certain methods sorted out the feature points in the attributes of the path to order and realign the videos captured by accomplishing the correspondence across the paths under surveillance this method termed as 
the "feature based alignment algorithm was developed by the author Singh et al [3] this operated by identifying the centroid of the objects that are moving followed by acquiring the transformation matrix. Further author Smistad et al [4] devised the selection process for the deciding the transformation matrix with the least MSE, and the author Bentley et al [5] repeated the same process mentioned above to attain an optimal transformation matrix.

The author Georgios D., et al [6] developed the methods to acquire the parameters for ordering the video by working on the spatial brightness variations and isolating them. The method builds the pyramid for the spatiotemporal and determines the each stages spatial-temporal ordering and the output at the current state is propagated to the following state using the initial determination.

The method with the an algorithm to develop an supplementary video using the basic video based on its spatial and temporal registration was developed using the author SG, Anuradha et al [7] and Kumar et al [8] the algorithm devised allowed to select the secondary images features points according to the weight function but was not successful because of the incoherence experienced in the analogous area. Rublee, et al [9] conducted "ORB: An efficient alternative to SIFT or SURF." J. Deva et al [10] proposed the smart transport facility with traffic signal sensors using the computer vision.

Manoharan et al [11] performed "Image Detection, Classification and Recognition for Leak Detection in Automobiles." The author Shakya, Subarna et al [12] conducted the "Virtual Restoration of Damaged Archeological Artifacts Obtained from Expeditions Using 3d Visualization." Further Kumar, R et al [13] presented the "novel report on architecture, protocols and applications in Internet of Things (IoT)."

\section{Proposed Design}

A standard algorithm for processing and ordering the videos properly was developed in the proffered mechanism, to provide accurate aligning and synchronization compared to the prevailing system such as Lucas-Kanade's. The architecture design presenting the overview of the proffered mechanisms activities is depicted in figure. 1 below. 
Journal of Innovative Image Processing (JIIP) (2020)

Vol.02/ No. 01

Pages: $25-34$

https://www.irojournals.com/iroiip/

DOI: https://doi.org/10.36548/jiip.2020.1.003

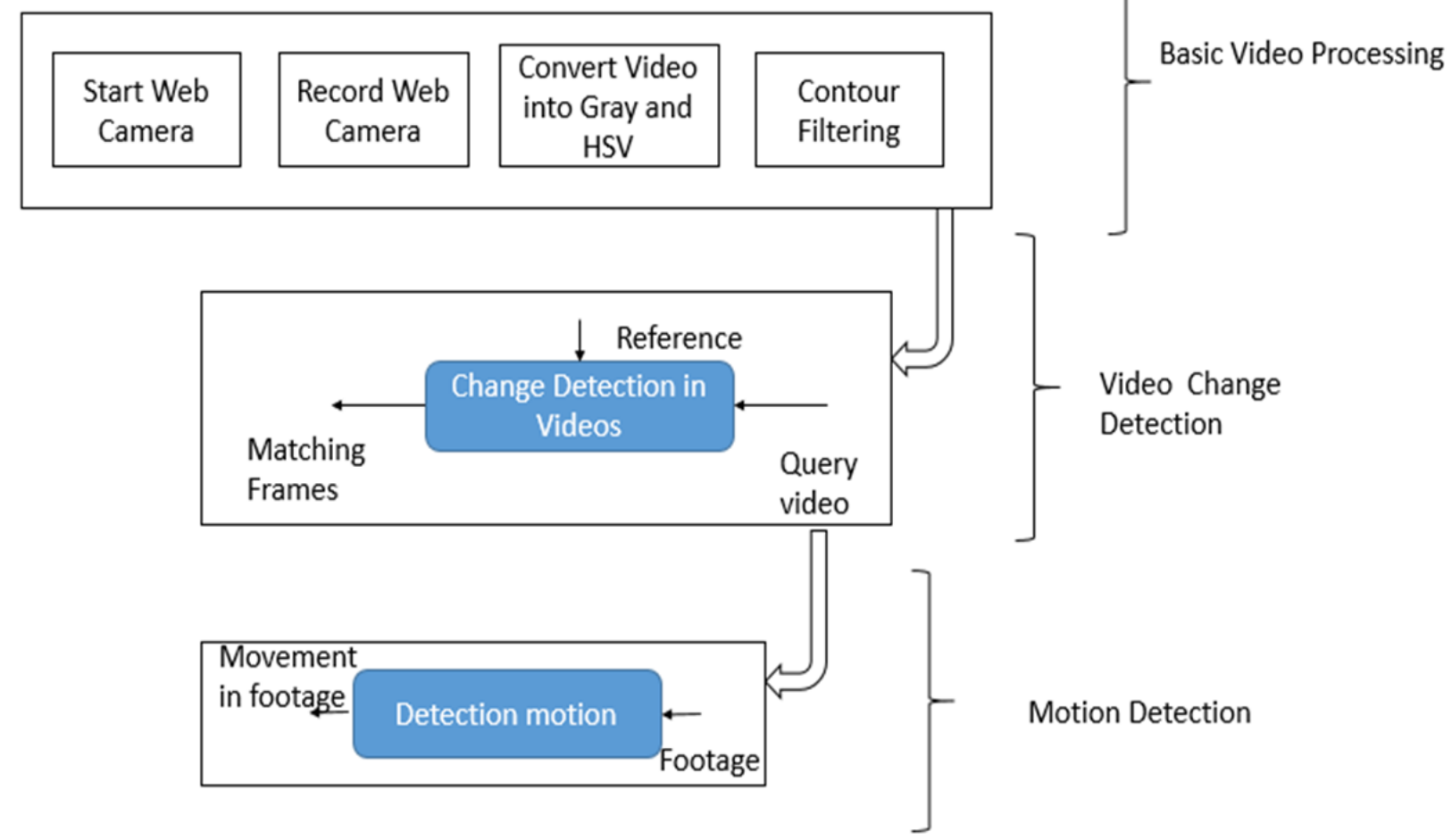

Figure .1 Proposed Model Overview

The architecture is encompassed with multiple layers, components and algorithms to achieve the proper ordering of the video, the architecture has a layer to access the data. This layer enables the admittance to all probable functions on the data base, the proposed method uses the MySQL data base. Every other layer communes with the layer of data access to satisfy the demands of their data access. The proposed system maintains the account details of the end user in the layer called account operations by following a set of procedures such as registration, login details, account deletion etc. the video ordering of the proposed method done using the comparing two videos of different time in similar path and identifying the optimal one. This performed in the video change detection layer, once the optimal frame is sorted out it computed to using the algorithm to detect the happenings in it. The contour filtering (CF) identifies the object based on the color according to the objects color fed as input. The input is fed to CF through the Web-Cam or by the image that is available previously. The algorithm for the $\mathrm{CF}$ for identifying the edges is used to sort out the shape of the images, the results from the CF are in Black and White Form. Further the movement identification is done by feeding the video captured to it, the movement in the source file provided are identified and the source file trail is fed as input to the element detecting the movement. The trail is mostly 
Journal of Innovative Image Processing (JIIP) (2020)

Vol.02/ No. 01

Pages: 25- 34

https://www.irojournals.com/iroiip/

DOI: https://doi.org/10.36548/jiip.2020.1.003

provided by the registered user who logs into the system. The flow chart below in figure. 2 provides the operation of the detecting changes in the video.

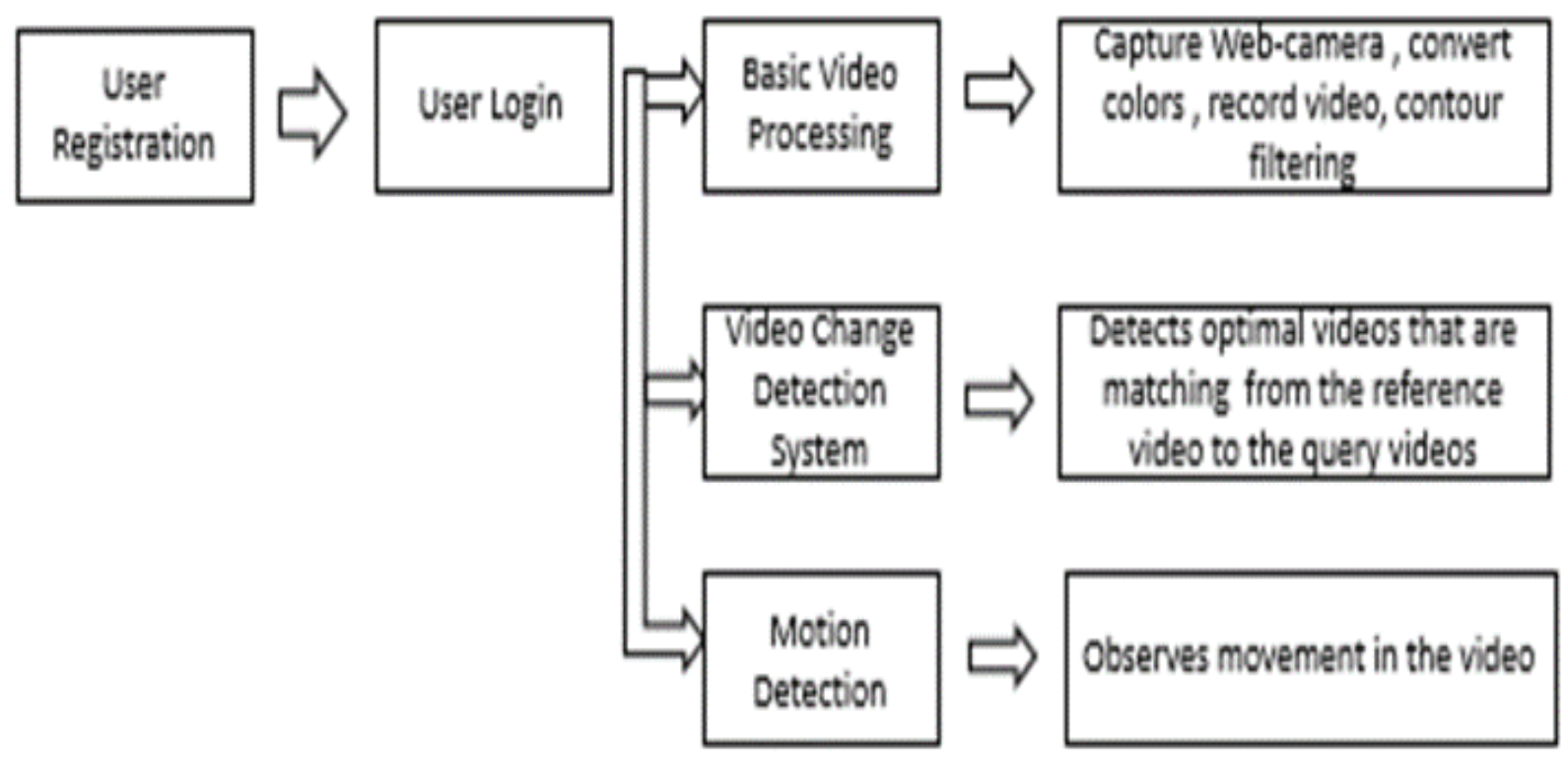

Figure.2Change Detection in Videos

The algorithm below in figure. 2 is the motion detection algorithm that identifies the movement and unprecedented activities in the public and also helps in traffic monitoring 
Journal of Innovative Image Processing (JIIP) (2020)

Vol.02/ No. 01

Pages: 25- 34

https://www.irojournals.com/iroiip/

DOI: https://doi.org/10.36548/jiip.2020.1.003

Input : video from web cam or video already available

Initialize path for video (compress video and store as local file

system)

Read the path

initialize motion detection

\{

Initialize motion panel

\{

Initialize recorder,

Set back ground color,

Set font

Start

\{

Run()

Initialize grabber with the web camera

Get Snap form grabber

Initialize JV motion Detector (initialize, image dimension, cog

point, previous and current image, difference in image)

\{

Assign Snap to Previous image

\}

\}

Get a snap

JV motion detection. Cal move

previous cog point $\leftarrow$ Current Cog Point

Current Cog Point $\leftarrow$ Cog point

find difference

If difference $>0$

Report Cog Changes, repaint ()

Else

Record snap

If completed

Stop

Else

Next snap

\}

\}

Figure .3 Motion Detection Algorithm 


\section{Perfromance Analysis}

The proposed mechnaism of CCTV surveillance with the proper synchornization and alignmnet of video for identifying the unprecedeted violence and for the traffic monitoring, was implemneted in Java and evaluated using the MATLAB, to check its accuray compared to the prevailing methods. The accuracy of the proposed ssytem was measured comparing the true positives, true negatives, fals positive and the false negatives measured.

In total of 500 video set gathered from busy road on the city, from morning till afternoon the vidoes were segregated as testing and the training and the optimal matched frames were selected, subjected to video chage detection and motion detection. Further the accuracy was detected based on the true positives and the false positives and the false negatives. the equation .1 below gives the comoutation method for measuring the accuracy

$$
\text { Accuracy }=t_{p}+t_{n} / t_{p}+t_{n}+f_{p}+f_{n}
$$

Where the $t_{p}=$ true positive, $t_{n}=$ true negative, $f_{p}=$ false positive and $f_{n}=$ false neagtive, any error in the detection of the motion was evaluated using the equation 2

$$
\text { Error }=1-\left(t_{p}+t_{n} / t_{p}+t_{n}+f_{p}+f_{n}\right)
$$

The results in the table. 1 below provides the parmaetric measures acquired based on the perfromance of the proposed mechanism in the motion and the change detection in the videos. The results are based on the videos obtained on different timings on the same palce on diffferent days in an week. 
Journal of Innovative Image Processing (JIIP) (2020)

Vol.02/ No. 01

Pages: 25- 34

https://www.irojournals.com/iroiip/

DOI: https://doi.org/10.36548/jiip.2020.1.003

\begin{tabular}{|c|c|c|c|c|c|c|c|c|c|c|c|c|c|c|c|c|c|c|c|c|c|c|c|c|c|c|c|c|c|c|c|c|c|c|c|}
\hline \multirow{2}{*}{$\begin{array}{l}\text { Parameter } \\
\text { s } \\
\text { Measurued }\end{array}$} & \multicolumn{5}{|c|}{ Day 1} & \multicolumn{5}{|c|}{ Day 2} & \multicolumn{5}{|c|}{ Day 3} & \multicolumn{5}{|c|}{ Day 4} & \multicolumn{5}{|c|}{ Day 5} & \multicolumn{5}{|c|}{$\operatorname{Day} 6$} & \multicolumn{5}{|c|}{ Day 7} \\
\hline & 1 & 2 & 3 & 4 & 5 & 1 & 2 & 3 & 4 & 5 & 1 & 2 & 3 & 4 & 5 & 1 & 2 & 3 & 4 & 5 & 1 & 2 & 3 & 4 & 5 & 1 & 2 & 3 & 4 & 5 & 1 & 2 & 3 & 4 & 5 \\
\hline $\begin{array}{l}\text { True } \\
\text { Positive }\end{array}$ & 35 & 22 & 30 & 23 & 21 & 25 & 26 & 27 & 30 & 29 & 34 & 32 & 31 & 30 & 22 & 21 & 20 & 27 & 29 & 30 & 33 & 35 & 32 & 33 & 25 & 26 & 21 & 20 & 24 & 25 & 27 & 28 & 45 & 30 & 40 \\
\hline $\begin{array}{l}\text { True } \\
\text { Negative }\end{array}$ & 20 & 15 & 13 & 10 & 11 & 12 & 14 & 13 & 12 & 10 & 17 & 15 & 19 & 10 & 9 & 15 & 13 & 11 & 12 & 16 & 13 & 14 & 15 & 14 & 16 & 17 & 18 & 20 & 13 & 15 & 16 & 13 & 14 & 15 & 12 \\
\hline $\begin{array}{l}\text { False } \\
\text { Positive }\end{array}$ & 3 & 4 & 5 & 6 & 7 & 2 & 1 & 4 & 6 & 4 & 7 & 3 & 2 & 4 & 5 & 7 & 4 & 7 & 5 & 3 & 2 & 10 & 8 & 9 & 7 & 4 & 5 & 7 & 8 & 9 & 5 & 6 & 5 & 3 & 5 \\
\hline $\begin{array}{l}\text { False } \\
\text { Negative }\end{array}$ & 2 & 2.1 & 2.3 & 3.1 & 1.1 & 1.0 & 1.5 & 2.5 & 1.8 & 1.9 & 2 & 1.5 & 1.7 & 2.2 & 2.6 & 27 & 3 & 2.1 & 3.4 & 2 & 3 & 1 & 2.5 & 3.2 & 3.1 & 3.3 & 2.7 & $\begin{array}{l}2 . \\
9\end{array}$ & 2.4 & 3.6 & 3.5 & 3.3 & 2.4 & 2.1 & 2 \\
\hline
\end{tabular}

Table.1 Performance Measure

The figure. 4 below shows the aaccuracy in the perfomance of the proposed model and it is compared to the accuracy in odering the videos in the Lucas-Kanade's, it was observed that proposed method showed good and better results than the prevailing. Based on the different set of videos observed on a day on different timing.

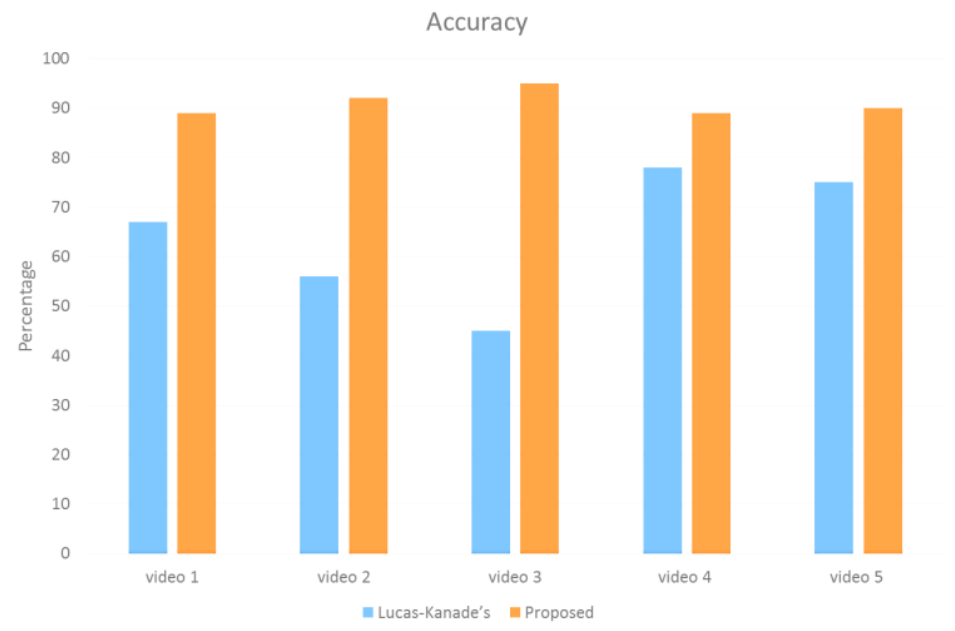

Figure.4 Accuracy in Ordering 
Journal of Innovative Image Processing (JIIP) (2020)

Vol.02/ No. 01

Pages: 25- 34

https://www.irojournals.com/iroiip/

DOI: https://doi.org/10.36548/jiip.2020.1.003

The figure. 5 blelow present the error rate observed in the propose method and the prevailing method the results shows the error rate observed in the proposed method was very low compared to the prevailing.

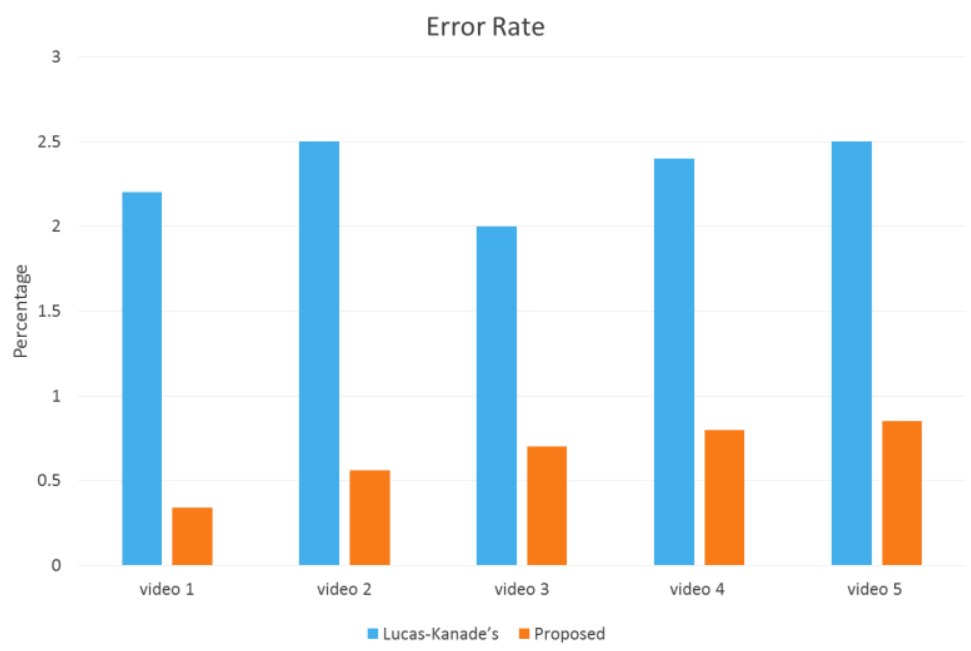

Figure.5 Error Rate

\section{Conclusion}

The paper developed an perfect alignment and synchornization method for the CCTV surveillance utilized in monitoring the traffic and the unprecendented violence. The synchorinization and the ordering was achived utilizing the change detection in the videos and detecting the movements. Once the matched frames were identified the acitivites in the video were identified. The proposed method was writtenin jave and executed in MATLAB and the accuracy $n$ ordering and synchornizing was verified, based on the measure of true positive, false positive, tue negative and true positive and the error rate was calculated. The accuraccy and the error rate was compared with the existing system to evince the performance upliftment.

\section{References}

[1] Bradski, Gary. "The opencv library." Dr Dobb's J. Software Tools 25 (2000): 120-125.

[2] Lowe, David G. "Object recognition from local scale-invariant features." In Proceedings of the seventh IEEE international conference on computer vision, vol. 2, pp. 1150-1157. Ieee, 1999. 
[3] Singh, Sanjay, A. S. Mandal, Chandra Shekhar, and Anil Vohra. "Real-time implementation of change detection for automated video surveillance system." ISRN Electronics 2013 (2013).

[4] Smistad, Erik, Thomas L. Falch, Mohammadmehdi Bozorgi, Anne C. Elster, and Frank Lindseth. "Medical image segmentation on GPUs-A comprehensive review." Medical image analysis 20, no. 1 (2015): 1-18.

[5] Bentley, Jon Louis. "Multidimensional binary search trees used for associative searching." Communications of the ACM 18, no. 9 (1975): 509-517.

[6] Evangelidis, Georgios D., and Emmanouil Z. Psarakis. "Parametric image alignment using enhanced correlation coefficient maximization." IEEE Transactions on Pattern Analysis and Machine Intelligence 30, no. 10 (2008): 1858-1865.

[7] SG, Anuradha, K. Karibasappa, and B. Eswar Reddy. "Video Segmentation For Moving Object Detection Using Local Change \& Entropy Based Adaptive Window Thresholding."

[8] Kumar, Rupesh, Sumana Gupta, and K. S. Venkatesh. "Cut scene change detection using spatio temporal video frame." In 2015 Third International Conference on Image Information Processing (ICIIP), pp. 474-479. IEEE, 2015.

[9] Rublee, Ethan, Vincent Rabaud, Kurt Konolige, and Gary Bradski. "ORB: An efficient alternative to SIFT or SURF." In 2011 International conference on computer vision, pp. 25642571. Ieee, 2011.

[10] Koresh, M. H., and J. Deva. "Computer vision based traffic sign sensing for smart transport." J. Innovative Image Process.(JIIP) 1, no. 01 (2019): 11-19.

[11] Manoharan, Samuel. "Image Detection, Classification and Recognition for Leak Detection In Automobiles." Journal of Innovative Image Processing (JIIP) 1, no. 02 (2019): 61-70.

[12] Shakya, Subarna. "Virtual Restoration Of Damaged Archeological Artifacts Obtained From Expeditions Using 3d Visualization." Journal of Innovative Image Processing (JIIP) 1, no. 02 (2019): 102-110.

[13] Kumar, R. Praveen, and S. Smys. "A novel report on architecture, protocols and applications in Internet of Things (IoT)." In 2018 2nd International Conference on Inventive Systems and control (ICISC), pp. 1156-1161. IEEE, 2018. 